\title{
Características do suicídio em Santa Catarina: um estudo do período de 2007 a 2016
}

Idonézia Collodel Benetti. Universidade Federal de Santa Catarina Leandro Ribeiro Molina. Secretaria Municipal de Saúde de Florianópolis Alan Kornin. Universidade Anhembi Morumbi/UAM

\section{Resumo}

A morte por suicídio é considerada uma epidemia global e um importante problema de saúde pública. O objetivo deste trabalho foi descrever as características epidemiológicas do suicídio no estado de Santa Catarina. Trata-se de um estudo ecológico, espaço-temporal e descritivo. Foi analisada uma série histórica de 10 anos, a partir de dados do Sistema de Notificação de Mortalidade do Ministério da Saúde, no período 2007-2016. Os cálculos por macrorregiões revelaram taxas mais elevadas no Oeste do estado. As taxas apresentaram-se três a quatro vezes maiores no sexo masculino, aumentando conforme a idade. Verificaram-se maiores proporções de ocorrência de suicídio em brancos e nos casados. Enforcamento foi o meio mais utilizado por ambos os sexos. As elevadas taxas de óbitos por autoextermínio, observadas neste estado da federação, chamam atenção para necessidade de aprimoramento de políticas públicas para prevenção do suicídio.

Palavras-chave: suicídio; causas externas; epidemiologia; saúde mental; saúde pública.

\begin{abstract}
Aspects of suicide in Santa Catarina: a study from 2007 to 2016. As silent events, suicide deaths are also considered a global epidemic and an important public health problem. The aim of this study was to describe the epidemiological characteristics of suicide in the Brazilian state of Santa Catarina. It is an ecological, spatial-temporal and descriptive study. Data from historical series of 10 years were analyzed from Mortality Reporting System of the Ministry of Health in the period 2007-2016. Calculations by macroregions revealed higher rates in the state's Western region. Suicide rates show up three to four times higher in males, increasing proportionally to age. There was greater occurrence of suicide proportions among whites and married. Hanging was the most common method used by both sexes, to put an end to life. The high death rates for self-extermination observed in this state call attention to necessity for improvement of public policy for suicide prevention.
\end{abstract}

Keywords: suicide; external causes; epidemiology; mental health; public health.

\section{Resumen}

Características de suicidio en Santa Catarina: un estudio de período de 2007 a 2016. La muerte por suicidio es considerada una epidemia global y un importante problema de salud pública. El objetivo del estudio fue describir las características epidemiológicas de suicidio en el estado de Santa Catarina (SC). Se trata de estudio ecológico, espacio-temporal y descriptivo. Se analizó serie histórica de 10 años a partir de datos de lo Sistema de Información de Mortalidad del Ministerio de la Salud en el período 2006-2017. Los cálculos por macrorregiones revelaron tasas más altas en el Oeste. Las tasas fueron tres a cuatro veces mayores en hombres, lo que aumenta con la edad. Fueron mayores las proporciones de ocurrencia de suicidio en blancos y casados. El ahorcamiento fue el método más utilizado por ambos sexos. Las altas tasas de mortalidad por autoexterminio observadas en esse estado llaman atención para necesidad de mejorar políticas públicas para prevención del suicidio.

Palabras clave: suicidio; causas externas; epidemiología; salud mental; salud pública. 
O estudo das taxas de suicídio é parte de uma herança importante do patrimônio deixado pela sociologia. Emile Durkheim tomou esta forma de comportamento como tema de investigação sociológica em seu livro clássico "Suicide" publicado em 1897. Esta obra apresenta a análise das variações em nível macro das taxas, tais como as diferenças entre as religiões católica e protestante na Europa ou variações por idade e sexo, em um esforço para mostrar como certas características das sociedades poderiam explicar o suicídio como um "fato social".

Etimologicamente o termo suicídio significa: "suis" = eu + "caedes" = ação de matar. Na literatura este evento é descrito como sendo um ato privado, intencional e voluntário de tirar a própria vida (Durkheim, 2013). Do ponto de vista da psicologia, o suicídio é considerado um ato de desespero causado pela exaustão total da vida, quando nada mais importa e a fuga para a morte é uma oportunidade para cessar o sofrimento, quando agentes externos - sociedade - projetam-se como torturadores (Cassorla, 2017). Na concepção da Organização Mundial da Saúde (OMS) (2003), o suicídio é um ato de pôr fim à própria vida, onde o executante tem conhecimento prévio do desfecho deste ato, estando ciente do resultado fatal.

O comportamento suicida, que atraiu atenção de Durkheim no século XIX, é hoje um importante problema de saúde pública em todo o mundo. Esse fenômeno multidimensional, oriundo da interação de fatores ambientais, sociais, genéticos, biológicos e fisiológicos (World Health Organization [WHO], 2015), é a segunda principal causa de morte em nível mundial entre pessoas jovens (15-29 anos de idade). A cada 40 segundos uma pessoa provoca morte intencional autoinfligida em algum lugar do mundo; a cada ano, mais de um milhão de pessoas dão fim à própria vida voluntariamente e entre 100 e 200 milhões de indivíduos se envolvem em tentativas de autoextermínio (Costanza et al., 2018).

O suicídio configura-se como uma questão delicada e, por esta razão, é muito provável que seja sub-relatada. Vale salientar que registrar um ato fatal de autolesão, envolvendo intenção de morte, é um procedimento complexo que envolve diversas autoridades e, em muitos casos, há a aplicação da lei o que, muitas vezes, acaba culpabilizando as pessoas que cercam esse evento fatídico. Para a maioria dos grupos sociais, os atos de suicídio são marcados pelo silêncio (o "não dito"), sendo rodeados por estigmas, tabus, preconceitos e constrangimentos, de forma que o assunto é quase sempre evitado ou proibido (Yehudit, 2018).

Assim, levando-se em consideração estes fatores, existe subnotificação da mortalidade por suicídio, que varia de acordo com a região e a cultura. Em países sem registros confiáveis, a morte por suicídios pode ser bem mais elevada do que se apresenta em números. Mesmo em países com bom registro vital de dados, esse fenômeno pode muitas vezes ser erroneamente classificado como um acidente ou outra causa de morte.

Vale realçar que, quando considerados os números absolutos, os eventos de suicídio superam os valores totais de mortes por homicídios e dos óbitos ocorridos em guerras, somados juntos. Embora mais de $79 \%$ dos suicídios globais tenham ocorrido em países de baixa e média renda em 2016, esse é um fenômeno, também, de lugares com posição privilegiada no ranking socioeconômico (lemmi et al., 2016; WHO, 2018).

No Brasil, a morte intencional autoinfligida é mais frequente na população branca e nas regiões mais ricas do país (Lima, 2017). No panorama mundial, em 1998 o suicídio representou 1,8\% da carga global de doenças e as estimativas para 2020 dão conta de que este número aumentará para 2,4\%. Na maioria dos países, que apresentam informações confiáveis, o suicídio está entre as 10 principais causas de morte em todas as idades (WHO, 2018).

Estima-se que 793.307 mortes por suicídio ocorreram em todo o mundo em 2016 -507.730 (homens) 285.577 (mulheres). A faixa etária compreendida entre 30-49 anos teve - em 2016 - o valor absoluto mais alto (245.316) e os jovens - 15 a 29 anos - tiveram o segundo maior valor - 212.275. Estima-se, também, que as mortes suicidas anuais globais podem subir para 1,5 milhões em 2020. Isso representa uma morte a cada 20 segundos e uma tentativa a cada dois segundos. As tentativas de suicídio são até 20 vezes mais frequentes do que suicídios consumados (WHO, 2018).

As taxas mais altas de suicídio são registradas em países do Leste Europeu - Lituânia (31,9), Rússia $(31,0)$ e Bielorrússia (26,2), na América do Sul (Guyana, 29,2) e na Ásia (Korea, 26.9) - dados de 2019 (World Population Review, 2019). É importante enfatizar que a Europa possui uma taxa média de 15,4 a cada 100.000 habitantes. No Brasil, as taxas de suicídio são consideradas baixas, variando entre 3,3 (1980) e 6,1 (2016), quando comparadas com os países previamente citados (WHO, 2018). Com estes dados, o que se pode observar é que, dentre os países que se encontram no topo do ranking para as taxas mais altas de suicídio, esse agravo ocorre em diferentes regiões do planeta e afeta pessoas de todas as nações, culturas e classes sociais. 
De fato, a literatura epidemiológica fornece evidências convincentes de que os países com as maiores taxas de suicídio do mundo são incrivelmente diversos. Enquanto na Europa as taxas vêm diminuindo 21,8 (2000) e 15,4 (2016) -, nas Américas o quadro apresenta movimento contrário - 8,3 (2000) e 9,8 (2016). Entretanto, é importante realçar que, das regiões constantes no quadro estatístico da WHO (2018), somente no continente americano houve aumento das taxas de suicídio, tanto para homens quanto para mulheres, quando verificada a tendência temporal entre os anos de 2000 a 2016. Entretanto, apesar do crescimento, o que se observa é que os valores apresentados estão abaixo da média global, que é de 10,6 por 100 mil habitantes (WHO, 2018).

Em 2016, o continente americano totalizou 97.288 óbitos por suicídio - 74.308 homens e 22.979 mulheres. Nesse mesmo mapa político, o Brasil é o $14^{\circ}$ país em número de suicídios e o quarto país latinoamericano com o maior crescimento no número absoluto de suicídios entre 2006 e 2015. Nesse período, o total de mortes autoinfligidas no país passou de 8.643 para 11.185 , o que representou um aumento de $33,6 \%$ (WHO, 2018). As taxas por 100 mil habitantes mostram que houve aumento de 5,3 (2000) para 6,2 (2016) um coeficiente de baixa magnitude, quando comparado aos resultados de outras regiões.

A morte intencional autoinfligida ocupa $3^{\circ}$ lugar entre os óbitos no Brasil. No ano de 2015 figurou em $1^{\circ}$ lugar entre pessoas na faixa etária compreendida entre 01 e 49 anos, com mais incidência no grupo jovem - 20 a 29 anos -, sendo a quarta causa de mortalidade por causas externas e a primeira causa de mortalidade em indígenas (Lima, 2017). Os homens, mais que as mulheres, são os que mais põem fim à vida, em todas as faixas etárias e em todas as Unidades da Federação.

Vale realçar, porém que, na condição de um país de grandes dimensões, as taxas de autoextermínio também diferem de região para região. Nesse aspecto, o sul do país se destaca por possuir índices acima da média nacional (Lovisi, Santos, Legay, Abelha, \& Valencia, 2009). Nesse cenário, o objetivo principal desta pesquisa foi descrever as características epidemiológicas do suicídio no estado de Santa Catarina (SC) no período entre 2007 e 2016.

\section{Método}

Trata-se de um estudo espaço-temporal, descritivo, caracterizando-se por uma pesquisa de levantamento de dados secundários, com taxas de mortalidade e proporções obtidas a partir das estatísticas de mortalidade publicadas pelo Sistema de Informação em Mortalidade (SIM) do Departamento de Informática do Sistema Único de Saúde (DATASUS, 2018), do Ministério da Saúde. Para a determinação do número de suicídios entre os anos 2007 e 2016 utilizou-se a décima revisão da Classificação Internacional de Doenças (CID-10), incluindo as categorias X60 a X84 (lesões autoprovocadas intencionalmente).

O período foi escolhido com o objetivo de se obter uma série histórica de uma década, sendo os dados do ano de 2016 os mais recentes disponibilizados pelo DATASUS no momento da coleta para esta pesquisa. Foram calculadas as taxas de mortalidade por suicídio: a) as gerais do estado e por municípios considerando média total do período, b) as bianuais por macrorregiões do estado, c) a média estadual, total e bianuais por faixa etária, e d) estaduais anuais por sexo - comparando estas últimas com as respectivas taxas padronizadas.

Para a padronização das taxas gerais de suicídio de SC, considerou-se como população padrão uma população composta pelo quantitativo populacional médio do estado no período analisado, distribuída de acordo com a estrutura etária da população-padrão da Organização Mundial da Saúde. As características epidemiológicas do suicídio foram descritas, segundo as variáveis envolvendo cor da pele, estado civil e método utilizado para dar fim à vida, a partir do cálculo de porcentagens proporcionais, levando em consideração o número total de óbitos.

\section{Resultados}

Considerando as taxas médias do período analisado (2007-2016), Santa Catarina é o segundo estado no ranking do suicídio no Brasil (8,62 óbitos/100 mil habitantes), ficando atrás apenas do Rio Grande do Sul (10,23 óbitos/100 mil habitantes). Além disso, os catarinenses também têm a quinta capital com maior número de suicídios no país (6,14 óbitos/100 mil 
habitantes) com coeficientes acima da média global para as capitais brasileiras que, no mesmo período, é de 4,23.

Na capital catarinense a taxa é menor do que a taxa do estado, indicando a tendência de predomínio de taxas mais elevadas no interior. A análise por cidades indicou que as taxas mais elevadas de morte autoinfligida estão localizadas nos municípios de Princesa (43,23 óbitos/ 100 mil habitantes) e Tigrinhos (34,41 óbitos/ 100 mil habitantes), ambos localizados na região Grande Oeste. A Tabela 1 mostra o ranking dos 20 municípios com maior taxa de suicídio no estado.

Tabela 1. Municípios com Taxas mais Elevadas de Mortalidade por Suicídio no Estado de Santa Catarina - Média do Período $2006-2017$

\begin{tabular}{|c|c|c|c|}
\hline Município & Macrorregião & Taxa/100 mil habitantes & Óbitos \\
\hline Princesa & Grande Oeste & 43,23 & 12 \\
\hline Tigrinhos & Grande Oeste & 34,41 & 6 \\
\hline Presidente Nereu & Vale do Itajaí & 30,44 & 7 \\
\hline Arabutã & Meio Oeste & 28,74 & 12 \\
\hline Iraceminha & Grande Oeste & 28,40 & 12 \\
\hline São Miguel da Boa Vista & Grande Oeste & 26,01 & 5 \\
\hline Santa Rosa de Lima & Sul & 23,88 & 5 \\
\hline Ipumirim & Meio Oeste & 23,12 & 17 \\
\hline Laurentino & Vale do Itajaí & 22,82 & 14 \\
\hline José Boiteux & Vale do Itajaí & 22,67 & 11 \\
\hline Lacerdópolis & Meio Oeste & 22,42 & 5 \\
\hline Santa Helena & Grande Oeste & 21,01 & 5 \\
\hline Lontras & Vale do Itajaí & 21,01 & 22 \\
\hline Anchieta & Grande Oeste & 20,64 & 13 \\
\hline Grão Pará & Sul & 20,58 & 13 \\
\hline Bandeirante & Grande Oeste & 20,57 & 6 \\
\hline Peritiba & Meio Oeste & 20,33 & 6 \\
\hline Lajeado Grande & Grande Oeste & 20,28 & 3 \\
\hline Guarujá do Sul & Grande Oeste & 20,17 & 10 \\
\hline Guabiruba & Vale do Itajaí & 19,77 & 38 \\
\hline
\end{tabular}

Fonte: MS/SVS/CGIAE - Sistema de Informações sobre Mortalidade - SIM.

Os cálculos realizados, segundo a divisão por macrorregiões, apresentam taxas mais elevadas nas regiões do Grande Oeste e do Meio Oeste, e as menores taxas nas regiões do Nordeste e na Grande Florianópolis. A Figura 1 apresenta as taxas médias bianuais das nove macrorregiões de saúde de Santa Catarina.

No que se refere ao sexo e faixa etária, como se pode observar nas Figuras 2 e 3 , as taxas de suicídio aumentam conforme a idade, porém apresentam-se três a quatro vezes maiores no sexo masculino. Para os homens, as taxas são ascendentes até a velhice. Para as para as mulheres, esta ascendência se estende até a faixa dos 50 anos, com posterior declínio.

A Figura 4 demonstra a variação das taxas de mortalidade em ambos os sexos, ao longo dos anos, e compara também com as taxas padronizadas.

Santa Catarina apresenta um percentual proporcional alto (90\%) de ocorrência de suicídio na população branca, se comparado à população de pardos (6\%) e negros (2\%). O estado civil das vítimas de suicídio responde por $39 \%$ das pessoas casadas e $33 \%$ das solteiras. Os separados judicialmente são $8 \%$ da população que optou pelo autoextermínio no período analisado. 




Biênio

2007-2008

2009-2010

$\square$ 2011-2012

$\square$ 2013-2014

$\square 2015-2016$

Figura 1. Suicídio nas Macrorregiões de Saúde no Estado de Santa Catarina - Médias Bianuais do Período 2007-2016.

Fonte: Elaborado pelos autores (2019)

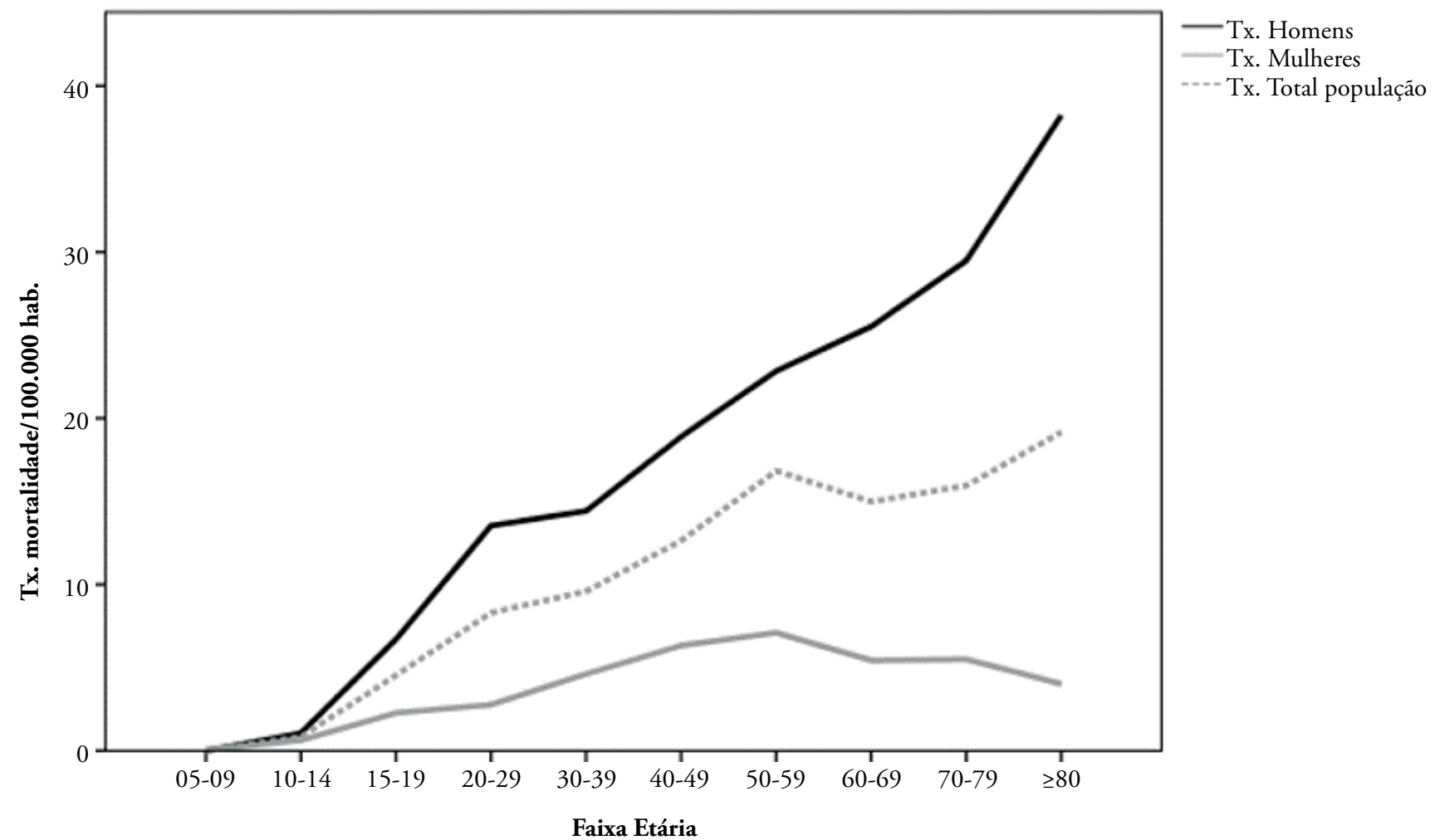

Figura 2. Taxa de Mortalidade por Suicídio em Relação a Sexo e Faixa Etária em Santa Catarina - Período 2007-2016.

Fonte: Elaborado pelos autores (2019) 




Biênio

2007-2008

$\square$ 2009-2010

$\square$ 2011-2012

$\square 2013-2014$

$\square$ 2015-2016

Figura 3. Suicídio em Santa Catarina conforme Faixa Etária - Médias Bianuais do Período 2007-2016. Fonte: Elaborado pelos autores (2019)

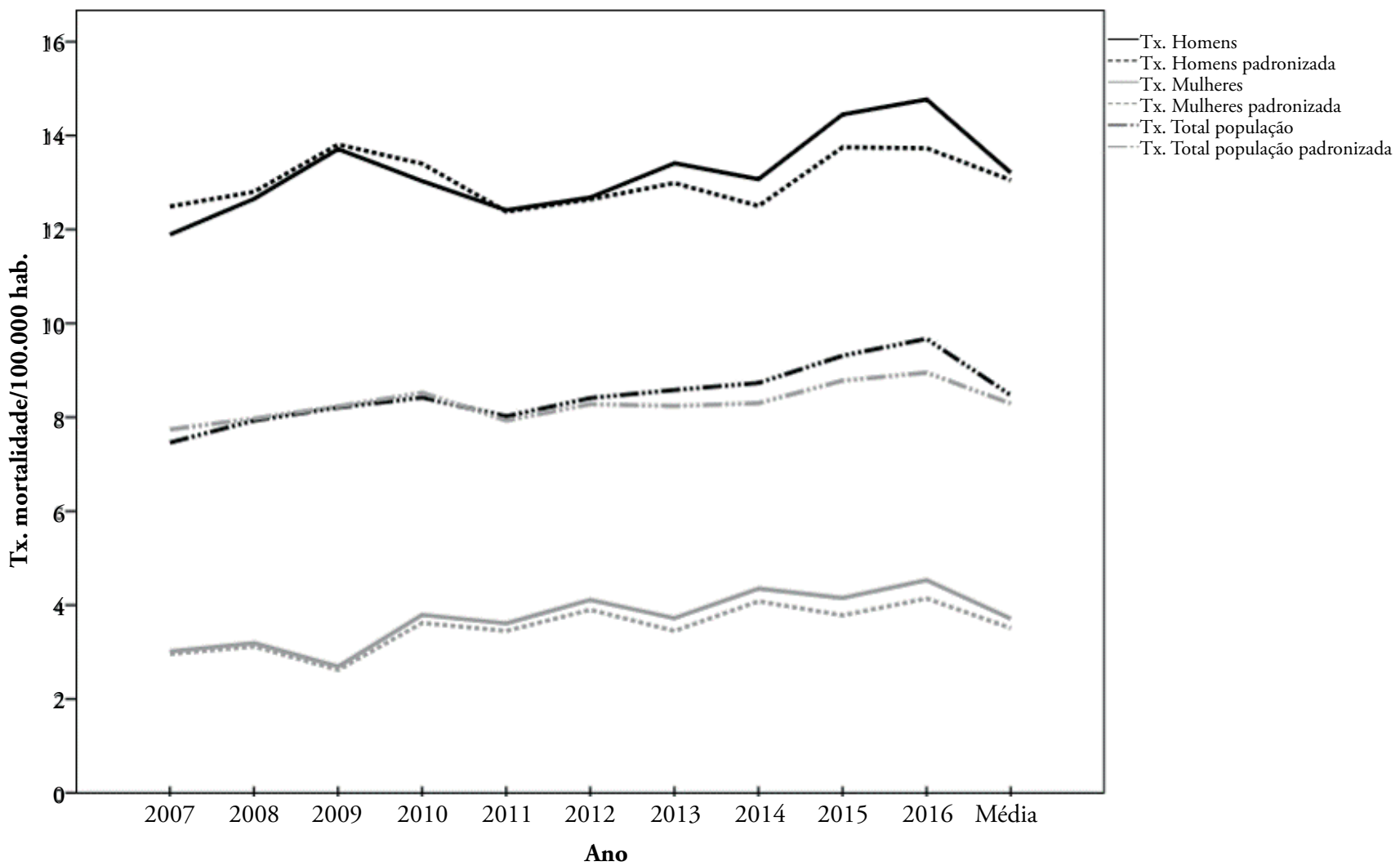

Figura 4. Mortalidade por Suicídio em Santa Catarina conforme Sexo - Período 2007-2016.

Fonte: Elaborado pelos autores (2019) 
Com relação aos métodos utilizados para tirar a vida, a maioria das vítimas preferiu o recurso do enforcamento/estrangulação/sufocação (categoria X70 na CID-10), considerado um meio tradicional nesse tipo de desfecho. O percentual para os homens nessa modalidade foi de $76 \%$ e para as mulheres $63 \%$. A segunda opção mais frequente para o sexo masculino foi a utilização de arma de fogo, correspondendo a $6 \%$ das mortes. O segundo, na ordem de preferência das mulheres foi o autoextermínio por uso de medicamentos e drogas, apresentando um percentual de $11 \%$.

\section{Discussão}

Os resultados globais (Sinyor, Tse, \& Pirkis, 2017) corroboram dados de outros estudos que apontam a região Sul do Brasil e Santa Catarina - com destaque para região Oeste - como detentores das taxas mais altas de suicídio no território nacional (Departamento de Vigilância Epidemológica [DIVE], 2017; Meneghel, Gomes, Faria, Carvalho, \& Falk, 2004; Peixoto \& Souza, 1999; Schmitt, Lang; Sehnem, \& Palosqui, 2014). Neste cenário, um dos achados mais consistentes na pesquisa sobre suicídio é que as mulheres fazem mais tentativas de pôr fim à vida do que os homens, mas os homens são mais propensos a morrer em suas tentativas do que as mulheres. Estudos focados nesse tema, em nível mundial, mostram que as mulheres estão 1,6 vezes mais propensas a tentar a morte; entretanto, são os homens que têm mais predisposição para a consolidação do ato, porque usam recursos mais letais (Franklin et al., 2017).

Entretanto, quando as taxas de suicídios efetivos entre os sexos são comparadas, homens se sobressaem em uma razão próxima de 4:1 (O'Connor et al., 2018; Sinyor et al., 2017). A diferença entre os eventos de suicídio, no que se refere aos sexos, pode ser explicada pelo fato de que os homens, por utilizarem métodos mais letais tais como armas de fogo e enforcamento, são mais efetivos do que as mulheres nas suas tentativas de pôr fim à vida; isso porque, predominantemente, elas escolhem as tentativas por envenenamento - e, geralmente, são socorridas a tempo em serviços de urgência e emergência, engrossando as estatísticas das tentativas e não as de suicídio consumado (Abreu, Silva, Kohlrausch, \& Soares, 2010). Então, restringir o acesso aos meios utilizados para cometer suicídio é um componente importante de estratégias globais de prevenção do desfecho. Em Santa Catarina, o enforcamento foi o meio mais utilizado, por ambos os sexos, para a intenção voluntária de tirar a própria vida. Esse achado está em sintonia com os dados apresentados por diversos estudos (Baptista \& Borges, 2005; Lovisi et al., 2009; Sehnem \& Palosqui, 2014), que apontam que esse método é o mais utilizado e o meio mais acessível de findar com a vida.

Observa-se, também, que o uso de armas de fogo está no ranking dos principais métodos de chegar ao óbito autoinfligido, comum aos homens. Esses dados são compatíveis com os apresentados pela WHO, quando se referem às taxas globais de suicídio (Sehnem \& Palosqui, 2014). Vale lembrar que esses recursos, além de serem tradicionais, são mais utilizados pelo sexo masculino, implicando em letalidade, o que sinaliza, também, que os dados catarinenses estão em harmonia com os registros mundiais no que se refere à expressividade da taxa de suicídios masculinos em relação ao feminino (Portella, 2013).

Fatores de risco comuns para comportamentos suicidas em ambos os sexos são transtorno mental anterior ao desfecho, abuso de substâncias e exposição à violência interpessoal. Em todo o mundo, tentativas de suicídio são comuns em indivíduos com Transtorno Depressivo Maior (Dong et al., 2018). Há uma ligação entre o comportamento suicida e uso e abuso de álcool e outras drogas. A aceitação de que essas condições de saúde mental são doenças, que necessitam de tratamento, deve ser promovida na população. O empenho na prevenção ao suicídio também é susceptível de ser reforçado pela contínua expansão do acesso ao tratamento eficaz para a depressão, abuso de álcool e outras drogas, ansiedade, e outros transtornos mentais. Nesse cenário, é vital que tais transtornos sejam identificados, e tratados, e se compreenda a dimensão dos problemas de saúde mental (Schneider, 2009).

Fatores de risco específicos para o sexo feminino incluem transtorno de estresse pós-traumático, transtorno bipolar, violência, sintomas depressivos, problemas interpessoais e aborto anterior; para o sexo masculino, a especificidade dos fatores é encontrada quando há problemas de comportamento/conduta disruptivos, falta de esperança, separação/divórcio dos pais, comportamento suicida do amigo e acesso a meios letais (Miranda-Mendizaba et al., 2019).

É interessante lembrar que as mulheres, mais que os homens, reconhecem precocemente sinais de risco para depressão, suicídio e doença mental e buscam auxílio em momentos de crise, ao participarem de redes de apoio social. O comportamento de se 
engajar na busca de ajuda, com disposição geral para falar sobre problemas emocionais, além de, frequentemente, buscar amigos e profissionais como fontes de ajuda, tem sido descrito na literatura (Cassorla, 2017; Liddon, Kingerlee, \& Barry, 2018).

Nas mulheres, as tentativas de suicídio são vistas como uma maneira de procurar apoio - um jeito de chamar a atenção e buscar auxílio (Freitas \& Borges, 2017; Liba et al., 2016). Talvez o fato de as mulheres fazerem este reconhecimento, e procurarem por ajuda, seja uma justificativa para a menor incidência de morte autoinfligida no sexo feminino, quando comparado ao suicídio consumado no sexo masculino. Nas mulheres também são encontrados comportamentos relacionados à baixa prevalência de alcoolismo, à religiosidade, e ao desempenho de variados papéis e funções durante a vida, considerados como indicadores protetivos para o suicídio (Meneghel et al., 2004).

Evidências preliminares sugerem que os homens deprimidos podem ser particularmente vulneráveis ao suicídio, em oposição a mulheres deprimidas (Nock \& Kessler, 2006). Em Santa Catarina, os resultados relativos aos homens remetem aos achados de Durkheim (2013), que apontam para o casamento como um mecanismo de proteção para o comportamento suicida. Na faixa etária adulta, quando há a maioria dos divórcios, a alienação parental (Mcclintock, 2014), a separação dos filhos, o afastamento e o ressentimento do pai que perdeu a guarda da criança (Kposowa, 2003), as perdas financeiras, e possíveis vazios existenciais, que levam à depressão (Yamamura, 2010), podem gerar o declínio da saúde mental dos homens. Esse quadro de fragilidade, além de deixá-los vulneráveis, pode promover a busca pela morte autoinfligida.

A presença de um transtorno mental é um dos mais importantes fatores de risco para o suicídio (Botega, 2015). Os transtornos mentais ocasionam problemas de ordem psicológica, que interferem na expectativa de vida das pessoas, mesmo que estas apresentem as melhores possibilidades de uma vida longa e melhor (Fegg, Kraus, Graw, \& Bausewein, 2016). Entretanto, é importante salientar que pacientes com longa duração de doença física e mental têm maior risco de cometer suicídio. Embora esse risco seja conhecido na doença mental, os profissionais devem estar cientes de que pacientes com doença física grave e crônica também apresentam risco para o autoextermínio.

No cenário mundial, as taxas de suicídio continuam a aumentar nos homens da faixa etária compreendida entre 60 e 94 anos (Apesoa-Varano, Barker, $\&$ Hinton, 2018; WHO, 2018). Os valores referentes ao suicídio por faixa etária e sexo em Santa Catarina estão em harmonia com as descrições da literatura: incidência três a quatro vezes maiores no sexo masculino, sendo que, para eles, os coeficientes aumentam conforme a idade (WHO, 2018). Ainda, há sugestões na literatura de que a rigidez no senso de self e as adequações, e o manejo para lidar com os acontecimentos da vida, podem ser disposições individuais que contribuem para a propensão ao suicídio de homens mais velhos.

Algumas dimensões de inflexibilidade mapeiam aspectos de scripts de hegemonia-masculinidade; um roteiro de masculinidade forte, independente, dominante, voltado para autonomia e controle, pode ameaçar não apenas o bem-estar dos homens, mas também sua sobrevivência, especialmente no final da vida (Canetto, 2017). Para os homens descendentes de europeus, facilmente encontrados em Santa Catarina, a rigidez no sentido de self pode ser reforçada por gênero e privilégio étnico, já que a vida deles costuma ser relativamente protegida de desafios para status, autonomia e controle, quando adultos. Tais privilégios tendem a mudanças com o declínio da idade (Canetto, 2017).

Homens com ascendência europeia, que rigidamente e tenazmente seguem os roteiros de masculinidade hegemônica, particularmente os roteiros independentes ou machistas, podem estar menos preparados para os desafios e vulnerabilidades do envelhecimento (Canetto, 2017; Winterrowd, Canetto, \& Benoit, 2017). Ainda, o suicídio em adultos idosos pode ser precipitado por problemas de saúde - doença grave, doença terminal ou necessidade de assistência domiciliar e também pode estar relacionado a outros estressores problemas financeiros, isolamento, solidão, ou morte de um cônjuge (Erlangsen, Stenager, \& Conwell, 2015; Winterrowd et al., 2017).

Jovens e adultos - homens e mulheres em idade economicamente produtiva - enfrentam crises financeiras, ou ajustes estruturais na economia, que resultam em desemprego, precarização do trabalho, contextos de superexploração, diminuição da renda pessoal e empobrecimento. Estes fatores têm sido correlacionados com o aumento do suicídio, particularmente em homens adultos, que sofrem o impacto das circunstâncias profissionais e financeiras, geradas por estressores econômicos (Cleary, 2012; Fraga, Massuquetti, \& Godoy, 2016).

Estudos realizados em Grécia e Espanha observaram a crise econômica como deflagradora de uma 
"epidemia" de suicídios. O aumento de refugiados e o impacto psicológico do crescimento da pobreza e do desemprego têm sido considerados como fatores importantes para suicídios em geral e para suicídios entre homens e mulheres (Davis, 2015; Ruiz-Perez, RodriguezBarranco, Rojas-Garcia, \& Mendoza-Garcia, 2017). Assim, além do desemprego, a desregulamentação, a flexibilização, a terceirização e a precarização do trabaIho em benefício do capital - e em detrimento do trabaIhador, gerando instabilidade financeira e pobreza são vistas como fatores predisponentes ao aumento das taxas de morte por autoextermínio.

Vale salientar que municípios com percentual mais alto de pessoas em situação de pobreza são os que apresentam os números mais elevados de morte por lesão autoinfligida (Fraga et al., 2016). Vale registrar que Santa Catarina é o estado da federação com o menor índice de pobreza registrado (Bagolin, Lopes, \& Vitcel, 2016). Entretanto, frente a momentos de crise, onde impera o desemprego, a deterioração econômica, a perda do poder de aquisição e do status social, mesmo que temporário, a pessoa pode ficar desestabilizada e ter sua autoestima afetada. Este cenário demanda a instalação de um quadro de ajustes compulsórios no estilo de vida, bem como de incerteza financeira e insegurança quanto ao futuro, além de preocupação e ansiedade. Nesse contexto, a honra aparece associada a perdas de bens, status e prejuízos nos empreendimentos; a reputação, em muitos casos fica maculada (Minayo, Meneghel, \& Cavalcante 2012).

Para os homens, o fator mais relevante, associado ao suicídio, é a perda do status e da estabilidade que o trabalho ou o emprego confere, fazendo emergir uma sensação de não pertencimento e ausência de lugar social que o aconchego do lar não consegue preencher (Minayo et al., 2012). Na esfera de gênero, masculinidade, competitividade e produtividade são conquistadas pela demonstração de força e o reconhecimento do caráter passa pela capacidade de ser provedor e acumular por meio do trabalho e do domínio que possui sobre a família e demais parceiros (Badinter, 1993; Connel \& Messerschimidt, 2013).

A depressão, o estresse, as cobranças para que se alcancem bens materiais, e um futuro profissional brilhante, tem custado muito caro a muitas pessoas. Condutas como a competitividade e a impulsividade, somadas ao acesso a meios letais, são consideradas como comportamentos que predispõem ao suicídio; um comportamento suicida anterior continua a ser um dos mais fortes preditores de um futuro comportamento suicida (Freitas \& Borges, 2017). Sendo assim, a população masculina, em idade economicamente produtiva, pode estar particularmente em risco de suicídio associado à crise financeira e pode se beneficiar de intervenções direcionadas para estes momentos.

Há algumas hipóteses que devem ser consideradas e aprofundadas em futuros estudos, para compreender melhor quais seriam os motivos que levam os homens a cometerem suicídio em idade economicamente ativa. Dentre algumas suposições, umas já mencionadas previamente, é possível que: haja resistência em procurar auxílio frente às dificuldades, em agendar consultas médicas ou psicológicas; exista a procura, mas há dificuldades em reconhecer ou expressar melhor os sentimentos, dificultando o diagnóstico pelos profissionais de saúde; alguns profissionais necessitem ser melhor capacitados para perceber, acolher e oferecer apoio imediato, e adequado, frente à demanda existente; haja a necessidade de que mais programas de educação permanente sejam desenhados, especificamente, para melhor preparar os profissionais da Atenção Primária à Saúde em relação à prevenção de quadros predisponentes ao suicídio.

Então, considerando que há uma alta prevalência de transtornos mentais entre os homens que morrem por suicídio, comportamentos de busca de ajuda e contato com o sistema de saúde podem diminuir o risco de morte por autoextermínio entre pessoas do sexo masculino (Miranda-Mendizabal et al., 2019). Esforços para diminuir as taxas de suicídio dos homens podem ser melhorados com um trabalho para desafiá-los a mudar a percepção sobre colocar ênfase no sucesso econômico, e ajudá-los a estender suas redes de apoio significativas (Sehnem \& Palosqui, 2014).

Com relação à faixa etária jovem, pensamentos e comportamentos de autolesão, na adolescência e juventude, estão associados à insatisfação com a imagem corporal (Claumann, Pinto, Silva, \& Pelegrini, 2018) e à ideação suicida (Cruz, Pascual, Salaberria, Echeburúa, \& 2015; Dooley, Fitzgerald, \& Giollabhui, 2015). Possivelmente, esta associação está relacionada a situações de constrangimento ou discriminação. Assim, perceber-se fora do peso ideal pode ser um fator de risco para desencadeamento do estresse e de sintomas de ansiedade.

Vale salientar que a insatisfação com a autoimagem, concomitante a outros estressores potenciais para esta faixa etária - desafios na área acadêmica, busca 
pela independência familiar, início dos relacionamentos amorosos, projetos para formar uma família, procura por estabilidade no emprego - podem favorecer um comportamento alimentar descontrolado (Roohafza, Sadeghi, Kabir, \& Aalaei, 2014) e pensamentos suicidas (Cho, Han, Kim, \& Lee, 2012). Então, se a ideação suicida pode levar a uma tentativa de morrer por autoextermínio, e esta última se constitui no principal fator de risco para a futura concretização do suicídio consumado (Freitas \& Borges, 2017), infere-se que a faixa etária adolescente e jovem está cercada por fatores que predispõem ao ato de, deliberadamente, cessar a vida.

No panorama global, um em cada nove jovens tentou suicídio e um em cada seis se envolveu em autolesão sem morte (O'Connor et al., 2018). Em território brasileiro, parece haver uma tendência do agravo na população adulta jovem, que começa mais cedo nas mulheres e se estende até os 50 anos. Esse quadro é coerente com os achados de Faria, Victora, Meneghel, Carvalho e Falk (2006), quando apontam uma correlação entre aumento das taxas de suicídio em mulheres agricultoras no Rio Grande do Sul. Dada a proximidade da região Oeste de Santa Catarina com aquele estado, é provável que haja semelhanças relativas a esse fenômeno nos dois estados, já que o Grande Oeste, um cluster de pequenas comunidades rurais, se caracteriza pela predominância da agricultura familiar.

Dadas as condições de desestímulo para viver e sobreviver da terra (Meneghel, \& Moura, 2018), aos camponeses restam: a) sujeição ao processo de desruralização, de desenlace e quebra de vínculos entre o trabalhador e a terra; b) expropriação; c) contemplação da agonia e a morte do campesinato; e d) exploração, patrocinada pela ocupação de grandes empresas, corporações e conglomerados industriais, que transformaram os processos de produção com a finalidade de ampliar o lucro e a mais-valia (Meneghel \& Moura, 2018).

Em um contexto de exploração do trabalho, não é difícil inferir que os arranjos do capitalismo, já previamente citados, operam para desestabilizar, emocionalmente, os trabalhadores, estimular a competitividade e a atribuição de culpabilidade pelo fracasso, instaurando inseguranças, quanto à permanência no emprego ou à perda da terra, que podem gerar patologias de medo, caracterizadas por angústia, mal-estar, depressão e ansiedades frente ao futuro. Tais agravos podem ser associados ao aumento de suicídios relacionados ao trabalho.
Ainda, no contexto do trabalho rural, há estudos que apontam possíveis relações entre a exposição aos transtornos mentais e aos agrotóxicos, o que pode contribuir para o aumento dos índices de suicídio (London, Flisher, Wesseling, Mergler, \& Kromhout, 2005). Apesar de possuir um território relativamente pequeno, Santa Catarina se caracteriza por uma ampla variedade étnica e cultural. No caso do Grande Oeste, observa-se que as taxas de suicídio por 100 mil habitantes são superiores às que respondem pelo litoral e pelo sul do estado.

Entretanto, o perfil étnico (descendentes de imigrantes europeus), presente na região Oeste do estado, também se encontra no Rio Grande do Sul, com as características de colonização semelhantes às encontradas em Santa Catarina (Faria et al., 2006). Porém, em se tratando de suicídio, um estudo descritivo desta natureza não tem poder para estabelecer se há relações causais em termos étnicos/culturais entre as diferentes colonizações e este tipo de óbito, o que dá margem para sugerir que esse é um assunto para futuros estudos.

\section{Considerações finais}

Um dos propósitos de se coletar dados epidemiológicos é o de criar e fornecer uma base para o desenvolvimento, priorização e estabelecimento de medidas de prevenção - procedimentos úteis na caracterização dos fenômenos investigados. Especificamente no caso dos suicídios, quanto mais informações houver, maiores serão as chances dos profissionais de saúde e da população auxiliarem na redução significativa de comportamentos de risco, incidência de tentativas e mortalidade por autoextermínio.

O comportamento suicida é um problema social e clínico extremamente relevante em algumas regiões do Brasil, como é o caso de Santa Catarina. Compreender as tendências do suicídio pode ajudar a identificar áreas geográficas de maior risco e concentrar esforços de prevenção. Nisso consiste a relevância desta investigação.

As elevadas taxas de óbitos por autoextermínio, observadas neste estado do sul do país, chamam atenção para necessidade de aprimoramento de políticas públicas para prevenção do suicídio, englobando toda população e setores da sociedade. Entretanto, com base em ensaios clínicos e experimentos naturais, de intervenções medicamentosas e psicoterapêuticas, melhorias na identificação e tratamento do paciente, alcance e restrição de métodos, há fortes evidências de que o suicídio é evitável. 
Os avanços contínuos na pesquisa sobre esse fenômeno estão enriquecendo as opções de abordagem para o manejo de pacientes suicidas. Maior sensibilidade ao potencial de comportamento suicida, melhorias contínuas na política de saúde pública, incluindo pesquisas básicas e translacionais, têm o potencial de contribuir para reduzir as taxas de morte por autoextermínio no futuro.

\section{Referências}

Abreu, K. P., Silva, M.D., Kohlrausch, E., \& Soares, J.F.(2010). Comportamento suicida: fatores de risco e intervenções preventivas. Revista Eletrônica de Enfermagem, 12(1), 195-200. doi: 10.5216/ree.v12i1.9537

Apesoa-Varano, E. C., Barker, J. C., \& Hinton, L. (2018). "If you were like me, you would consider it too": Suicide, older men, and masculinity. Society and Mental Health, 8(2), 157-173. doi: 10.1177\%2F2156869317725890

Badinter, E. (1993). XY: sobre a identidade masculina. Rio de Janeiro: Nova Fronteira.

Bagolin, I. P., Lopes, J. D., \& Vitcel, M. S. (2016). Pertinência, limites e desafios da adoção de linhas de pobreza absoluta ou relativa no Brasil e seus Estados. Espacios, 37(9), 19-32. Recuperado de https://www.revistaespacios.com/a16v37n09/16370919.html

Baptista, M. N., \& Borges, A. (2005). Suicídio: aspectos epidemiológicos em Limeira e adjacências no período de 1998 a 2002. Estudos de Psicologia, 22(4), 425-431. doi: 10.1590/S0103-166X2005000400010

Botega, N. J. (2015). Crise suicida: avaliação e manejo. Porto Alegre: Artmed.

Canetto, S. S. (2017). Suicide: Why Are Older Men So Vulnerable? Men and Masculinities, 20(1), 49-70. doi.org/10.1177/1097184X15613832

Cassorla, R. M. S. (2017). Suicídio. Fatores inconscientes e aspectos socioculturais: uma introdução. São Paulo: Blucher.

Cho, J. H., Han, S. N., Kim, J. H., \& Lee, H. M. (2012). Body image distortion in fifth and sixth grade students may lead to stress, depression, and undesirable dieting behavior. Nutrition Research and Practice, 6(2), 175-81. doi: 10.4162/nrp.2012.6.2.175

Claumann, G. S., Pinto, A. A., Silva, D. A. S., \& Pelegrini, A. (2018). Prevalência de pensamentos e comportamentos suicidas e associação com a insatisfação corporal em adolescentes. Jornal Brasileiro de Psiquiatria, 67(1), 3-9. doi: 10.1590/0047-2085000000177

Cleary, A. (2012). Suicidal action, emotional expression and the performance of masculinities. Social Science \& Medicine; 74(4), 498-505. doi: 10.1016/j.socscimed.2011.08.002

Connel, R. W., \& Messerschimidt, J. W. (2013). Masculinidade hegemônica:repensando o conceito. Estudos Feministas, 21(1), 241-282. doi: 10.1590/S0104-026X2013000100014

Costanza, A., Ambrosetti, J., Wyss, K., Bondolfi, G., Sarasin, F., \& Khan, R. (2018). Prévenir le suicide aux urgences: de la Théorie Interpersonnelle du Suicide à la connectedness. Revue Médicale Suisse, 14(593), 335-338. Recuperado de https://www.revmed.ch/ RMS/2018/RMS-N-593/Prevenir-le-suicide-aux-urgences-de-laTheorie-Interpersonnelle-du-Suicide-a-la-connectedness

Cruz, S., S., Pascual, A., Salaberria, K., \& Echeburúa, E. (2015). Normal weight and overweight female adolescents with and without extreme weight control behaviours: Emotional distress and body image concerns. Journal of Health Psychology, 20(6), 730-40. doi: $10.1177 \% 2 \mathrm{~F} 1359105315580214$

Davis, E. (2015). We've toiled without end": Publicity, crisis, and the suicide "epidemic" in Greece. Comparative Studies in Society and History, 57(4), 1007-103. doi: 10.1017/S0010417515000420

Departamento de Informática e Informação do Ministério da Saúde. (2018). Informações de Saúde. Recuperado de www.datasus.gov.br

Departamento de Vigilância Epidemiológica. (2017, 14 de setembro).Em apoio ao movimento Setembro Amarelo, Saúde divulga o panorama do suicídio em Santa Catarina. Recuperado de http://www.dive.sc.gov. br/index.php/arquivo-noticias/592-em-apoio-ao-movimento-setembroamarelo-saude-divulga-o-panorama-do-suicidio-em-santa-catarina

Dong, M., Zeng, L., Lu, L., Li, X., Ungvari, G., Ng, C., ... Xiang, Y. (2018). Prevalence of suicide attempt in individuals with major depressive disorder: A meta-analysis of observational surveys. Psychological Medicine, 49(10), 1691-1704. doi: 10.1017/S0033291718002301

Dooley, B., Fitzgerald, A., \& Giollabhui, N. M. (2015). The risk and protective factors associated with depression and anxiety in a national sample of Irish adolescents. Irish Journal of Psychological Medicine, 32(1), 93-105. doi: 10.1017/ipm.2014.83

Durkheim, E. (2013). As regras do Método Sociológico. São Paulo: Martins Fontes.

Erlangsen, A., Stenager, E., \& Conwell, Y. (2015). Physical diseases as predictors of suicide in older adults: a nationwide, register-based cohort study. Social Psychiatry and Psychiatric Epidemiology, 50(9), 1427-1439. doi: 10.1007/s00127-015-1051-0

Faria, N.X., Victora, C., Meneghel, S. N., Carvalho, A., \& Falk, J.W. (2006). Suicide rates in the state of Rio Grande do Sul, Brazil: Association with socioeconomic, cultural and agricultural factors. Cadernos de Saúde Pública, 22(12), 2611-2621 . doi: 10.1590/S0102-311X2006001200011

Fegg, M., Kraus, S., Graw, M., \& Bausewein, C. (2016). Physical compared to mental diseases as reasons for committing suicide: A retrospective study. BMC Palliative Care, 15(1). doi: 10.1186/s12904-016-0088-5

Fraga, W.S., Massuquetti, A., \& Godoy, M. R. (2016). Os determinantes socioeconômicos do suicídio: um estudo para os Municípios do Brasil e do Rio Grande do Sul. Revista Econômica, 18(2), 1-37. doi: 10.22409/economica.18i2.p300

Franklin, J. C., Ribeiro, J. D., Fox, K. R., Bentley, K. H., Kleiman, E. M., Huang, X., ... Nock, M. K. (2017). Risk factors for suicidal thoughts and behaviors: A meta-analysis of 50 years of research. Psychological Bulletin, 143(2), 187-232. doi: 10.1037/bul0000084

Freitas, A. P. A., \& Borges, L. M. (2017). Do acolhimento ao encaminhamento: o atendimento às tentativas de suicídio nos contextos hospitalares. Estudos de Psicologia, 22(1), 50-60. doi: 10.22491/1678-4669.20170006

lemmi, V., Bantjes, J., Coast, E., Channer, K., Leone, T., McDaid, D, ... Crick, L. (2016). Suicide and poverty in low income and middle-income countries: A systematic review. The Lancet Psychiatry, 3(8), 774-783. doi: 10.1016/S2215-0366(16)30066-9

Kposowa, A. (2003). Divorce and suicide risk. Journal of Epidemiology and Community Health, 57(12), 993-995. doi: 10.1136/jech.57.12.993

Liba, Y., Lemes, A. G., Oliveira, P. R., Nascimento, V. F., Fonseca, P. I. M. N., Volpato, R., \& Cardoso, T.P. (2016). Percepções dos profissionais de enfermagem sobre o paciente pós-tentativa de suicídio. Journal Health NPEPS, 1(1), 109-121. Recuperado de https://periodicos. unemat.br/index.php/jhnpeps/article/view/1437

Liddon, L., Kingerlee, R., \& Barry, J. A. (2018). Gender differences in preferences for psychological treatment, coping strategies, and 
triggers to help-seeking. The British Journal of Clinical Psychology, 57(1) 42-58. doi: 10.1111/bjc. 12147

Lima, C. M. (2017).Cenário epidemiológico do suicídio no Brasil. Ministério da Saúde Secretaria de Vigilância em Saúde. Departamento de Vigilância de Doenças e Agravos não Transmissíveis e Promoção da Saúde. Recuperado de http://legis.senado.leg.br/sdleg-getter/ documento/download/66128d2a-5fde-4061-8f2e-a705861940d9

London, L., Flisher, A. J., Wesseling, C., Mergler, D., \& Kromhout, H. (2005). Suicide and exposure to organophosphate insecticides: cause or effect? American Journal of Industrial Medicine, 47(4), 308-321. doi. https://doi.org/10.1002/ajim.20147

Lovisi, G. M., Santos, A. S., Legay, L., Abelha, L., \& Valencia, E. (2009). Análise epidemiológica do suicídio no Brasil entre 1980 e 2006. Revista Brasileira de Psiquiatria, 31(Supl. 2), S86-S93. doi: 10.1590/ S1516-44462009000600007

Mcclintock, E. (2014, dezembro). Why breakups are actually tougher on men. Psychology Today. Recuperado de https://www.psychologytoday. $\mathrm{com} / \mathrm{intl} / \mathrm{blog} / \mathrm{it}-\mathrm{s}$-man-s-and-woman-s-world/201412/ why-breakups-are-actually-tougher-men?tr=HdrQuote

Meneghel, S. N., Gomes V., C., Xavier F., Carvalho, L. A., \& Falk, J. W. (2004). Características epidemiológicas do suicídio no Rio Grande do Sul. Revista de Saúde Pública, 38(6), 804-810. doi: 10.1590/ S0034-89102004000600008

Meneghel, S. N., \& Moura, R. (2018). Suicídio, cultura e trabalho em município de colonização alemã no sul do Brasil. Interface, 22(67), 1135-46. doi: 10.1590/1807-57622017.0269

Minayo, M. C. S., Meneghel, S. N., \& Cavalcante, F. G. (2012). Suicídio de homens idosos no Brasil. Ciência \& Saúde Coletiva, 17(10), 2665-2674. doi: 10.1590/S1413-81232012001000016

Miranda-Mendizabal, A., Castellví, P., Parés-Badell, O., Alayo, I., Almenara, J., Alonso, I., ... Alonso, J. (2019). Gender differences in suicidal behavior in adolescents and young adults: Systematic review and meta-analysis of longitudinal studies. International Journal of Public Health, 1-19. doi: 10.1007/s00038-018-1196-1

Nock, M., \& Kessler, R. (2006). Prevalence of and risk factors for suicide attempts versus suicide gestures: Analysis of the National Comorbidity Survey. Journal of Abnormal Psychology, 115(3), 616-623. doi: 10.1037/0021-843X.115.3.616

O'Connor, R., Wetherall, K., Cleare, S., Eschle, S., Drummond, J., Ferguson, E., ... O'Carroll, R. (2018). Suicide attempts and non-suicidal self-harm: National prevalence study of young adults. BJPsych Open, 4(3), 142-148. doi: 10.1192/bjo.2018.14

Organização Mundial da Saúde (2003). Classificação de transtornos mentais e do comportamento da CID-10. Porto alegre: Artmed.

Peixoto, H. G.; \& Souza, M. L. (1999). O indicador anos potenciais de vida perdidos e a ordenação das causas de morte em Santa Catarina 1995. Informe Epidemiológico do SUS, 8(1), 17-25. doi: 10.5123/S0104-16731999000100003

Portella, C. H. (2013). Epidemiological profile of suicide in the Santa Catarina Coal Mining Region from 1980 to 2007. Trends in Psychiatry and Psychotherapy, 35(2), 128-133. doi: 10.1590/S2237-60892013000200006

Roohafza, H., Sadeghim M., Kabir, A., \& Aalaei, A. (2014). Effect of psychological distress on weight concern and weight control behaviors. Archives of Iranian Medicine, 17(9), 608-612. doi: 0141709/AIM.005

Ruiz-Perez, I., Rodriguez-Barranco, M., Rojas-Garcia A., \& MendozaGarcia, O. (2017). Economic crisis and suicides in Spain.
Socio-demographic and regional variability. The European Journal of Health Economics, 18(3), 313-320. doi: 10.1007/s10198-016-0774-5

Schmitt, R., Lang, M. G., Quevedo, J., \& Colombo, T. (2008). Perfi epidemiológico do suicídio no extremo oeste do estado de Santa Catarina, Brasil. Revista de Psiquiatria do Rio Grande do Sul, 30(2), 115-123. doi: 10.1590/S0101-81082008000300007

Schneider, B. (2009). Substance use disorders and risk for completed suicide. Archives of Suicide Research, 13(4), 303-316. doi: 10.1080/13811110903263191

Sehnem, S. B., \& Palosqui, V. (2014). Características epidemiológicas do suicídio no estado de Santa Catarina. Fractal: Revista de Psicologia, 26(2), 365-378. doi: 10.1590/1984-0292/624

Sinyor, M., Tse, R., \& Pirkis J. (2017). Global trends in suicide epidemiology. Current Opinion in Psychiatry, 30(1), 1-6. doi: 10.1097/YCO.0000000000000296

Winterrowd, E., Canetto, S. S., \& Benoit, K. (2017). Permissive beliefs and attitudes about older adult suicide: A suicide enabling script? Aging \& Mental Health, 21(2), 173-181. doi: 10.1080/13607863.2015.1099609

World Health Organization. (2015). World report on violence and health Genebra: World Health Organization.

World Health Organization. (2018). Suicide: Key Facts. Genebra: World Health Organization.

World Population Review. (2019). Suicide rate by country 2019 Recuperado de http://worldpopulation review.com/countries/ suicide-rate-by-country/

Yamamura, E. (2010). The different impacts of socio-economic factors on suicide between males and females. Applied Economics Letters, 17(10), 1009-1012. doi: 10.1080/13504850802676199

Yehudit, S. (2018). Choosing to enter the darkness - a researcher's reflection on working with suicide survivors: A collage of words and images. Qualitative Research in Psychology, 1-12. doi: 10.1080/14780887.2018.1442766

Idonézia Collodel Benetti, Doutora em Saúde Coletiva pela Universidade Federal de Santa Catarina, é Pós-doutoranda na mesma instituição. Endereço para correspondência: Campus Reitor João David Ferreira Lima, Rua Delfino Conti, s/n, Bloco H CEP: 88040-900. E-mail: idonezia@hotmail.com

Leandro Ribeiro Molina, Mestre em Saúde Coletiva pela Universidade Federal de Santa Catarina, é farmacêutico na Secretaria Municipal de Saúde de Florianópolis. E-mail: le_molina@hotmail.com

Alan Kornin, Mestre em Saúde Coletiva pela Universidade Federal de Santa Catarina, é docente na Universidade Anhembi Morumbi. E-mail: alankornin@gmail.com

Recebido em 29.nov.2016 Revisado em 27.fev.2019 Aceito em 10.jul.2019 